\title{
Is There Savings for Pavlovian Fear Conditioning after Neurotoxic Basolateral Amygdala Lesions in Rats?
}

\author{
Stephen Maren \\ Department of Psychology and Neuroscience Program, University of Michigan, \\ 525 E. University Avenue, Ann Arbor, Michigan 48109-1109
}

\begin{abstract}
Considerable evidence indicates an important role for amygdaloid nuclei in both the acquisition and expression of Pavlovian fear conditioning. Recent reports from my laboratory have focused on the impact of neurotoxic lesions of the basolateral complex of the amygdala (BLA) on conditional freezing behavior in rats. In these studies, I have observed severe effects of posttraining BLA lesions on the expression of conditional freezing even after extensive presurgical overtraining (25-75 trials). Moreover, I have found no evidence for sparing of fear memory (i.e., savings) in these rats when I assess their rate of reacquisition relative to BLA rats receiving minimal training ( 1 trial). In these experiments, freezing behavior was assessed using a conventional time-sampling procedure and expressed as a response probability. Although this measure is well established in the literature, it is conceivable that it is not sensitive to spared memory in rats with BLA lesions. To address this issue, I present a more detailed analysis of freezing behavior that quantifies latency to freeze, the number of freezing bouts, the duration of freezing bouts, and the probability distribution of bout lengths. I also include control data from untrained (noshock) rats. Consistent with my earlier reports, I find no evidence of savings of fear memory in rats with neurotoxic BLA lesions using several measures of freezing behavior. These results reiterate the conclusion that fear memory, as it is expressed in freezing behavior, requires neurons in the BLA. 1001 Academic Press
\end{abstract}

Key Words: amygdala; NMDA; lesion; overtraining; memory; savings conditioning; freezing; fear; rat.

\section{INTRODUCTION}

Fear is an adaptive emotion that is evoked by stimuli that signal danger. Among other functions, fear serves to both organize somatic and autonomic responses (e.g., freezing

This work was supported by grants from the National Institute of Mental Health (R29MH57865) and the University of Michigan. I thank Ki Goosens and Kevin Corcoran for helpful comments on an earlier draft.

Address correspondence and reprint requests to Stephen Maren, Department of Psychology, University of Michigan, 525 E. University Ave., Ann Arbor, MI, 48109-1109. Fax: (734) 763-7480. E-mail: maren@ umich.edu. 
and sympathetic arousal) and motivate action (e.g., escape and avoidance) in the face of threat. It has been recognized for decades that the amygdala is critically involved in the regulation of fear in mammals. Indeed, a large body of literature indicates that forms of learning that are motivated by fear, such as Pavlovian fear conditioning and instrumental avoidance conditioning, involve the basolateral complex of the amygdala (BLA; Davis, 1997; Fendt \& Fanselow, 1999; LeDoux, 2000; Maren, 2001; McGaugh, 2000).

Despite general agreement that the BLA has an important role in fear-motivated learning, considerable debate has emerged in recent years concerning the precise role of the amygdala in emotional learning and memory. On the one hand, many investigators have argued that neurons in the BLA play an enduring role in the encoding, storage, and retrieval of fear memories, particularly in Pavlovian conditioning paradigms (Davis, 1997; Fanselow \& LeDoux, 1999; Maren, 1999a). Alternatively, it has been argued that the amygdala plays only a temporary role in the consolidation of aversive memories and that these memories are not encoded within amygdaloid circuitry (Cahill \& McGaugh, 1998; Cahill, Weinberger, Roozendaal, \& McGaugh, 1999; Weinberger, 1998). These views are not mutually exclusive, and it is conceivable that the amygdala mediates both local storage and remote consolidation during aversive learning.

One approach to testing the view that the BLA is a locus for fear memory storage is to assess whether there is sparing of fear memory or "savings" in rats with amygdala dysfunction. Several studies using instrumental tasks, such as escape conditioning in rats, have reported savings after lesions of the amygdala (e.g., Parent, Tomaz, \& McGaugh, 1992). However, spared memory in avoidance or escape paradigms may be mediated by extraamygdaloid circuitry underlying the instrumental response, rather than savings of the associative fear memory per se. To understand this issue more fully, then, it is important to examine savings in a behavioral paradigm, such as Pavlovian fear conditioning, without an instrumental component.

To this end, Cahill and colleagues (2000) have recently examined the influence of pretraining lesions of the BLA on savings of contextual fear conditioning using freezing as a measure of fear. They found that rats with neurotoxic BLA lesions exhibited more contextual freezing after a footshock was delivered in a context where they had already received two footshocks ( $24 \mathrm{~h}$ earlier) than BLA rats that had received this earlier training in a different context (Cahill, Vazdarjanova, \& Setlow, 2000). Nonetheless, conditional freezing in rats with BLA lesions was greatly impaired relative to intact controls. These findings are consistent with other reports demonstrating the capacity for contextual fear conditioning in rats with neurotoxic BLA lesions (Maren, 1999b). However, savings in rats trained with BLA lesions may be mediated by neural systems that are not normally involved in the acquisition of fear memory in intact animals. Therefore, it is critical to address whether the BLA is a repository for Pavlovian fear memories in intact rats.

I have addressed this question by assessing Pavlovian fear conditioning in rats with posttraining BLA lesions. In these studies, I have found no evidence for savings of fear memory, at least as it is indexed by freezing behavior (Maren, 1998, 1999b), in rats with BLA lesions. Specifically, neurotoxic BLA lesions made 1 day after fear conditioning eliminated the expression of conditional freezing, and this effect was independent of the amount of initial training ( 1 or 75 conditioning trials). Moreover, the amount of original training did not affect the rate of reacquisition in rats with BLA lesions; BLA rats trained with 75 conditioning trials reacquired at the same rate as BLA rats trained with a single 
trial. In other words, BLA lesions made after training eliminated the savings for fear memory that is observed in rats with pretraining BLA lesions. Interestingly, posttraining amygdala lesions centered on the central nucleus that spare the BLA yield savings of memory for fear-potentiated startle (Kim \& Davis, 1993). This indicates that damage to the BLA itself is required to eliminate savings of Pavlovian fear memories.

Collectively, these data suggest that the memories for Pavlovian fear conditioning are formed and stored in the BLA of intact rats, insofar as there was no evidence for savings (at least as indexed by conditional freezing) in rats with posttraining lesions. However, it is possible that rats with BLA lesions exhibit savings relative to naive (nonshocked) rats upon additional training. Moreover, it is possible that other measures of freezing behavior, such as latency to freeze, might reveal spared performance in rats with posttraining BLA lesions. Therefore, the purpose of the present study was to (1) retrospectively analyze previously published data on the impact of posttraining BLA lesions on conditional freezing (i.e., Maren, 1998, 1999b) using a fine-grained analysis of freezing behavior and (2) compare savings in rats with BLA lesions to a naive (unshocked) control group. The goal of these analyses is to further explore the possibility that savings of Pavlovian fear memory, as it is expressed in freezing behavior, is evident in rats with posttraining BLA lesions.

\section{MATERIALS AND METHODS}

Subjects. The subjects were 112 adult male Long-Evans rats (200-224 g) obtained from a commercial supplier (Harlan Sprague-Dawley, Indianapolis, IN). After arrival, the rats were individually housed in standard stainless steel hanging cages on a 14:10-h light-dark cycle (lights on at 7:00 AM) and were provided free access to food and tap water. After housing, the rats were handled daily (10-20 s per rat) for 5 days to acclimate them to the experimenter. Some of the data from 60 of these subjects appeared in previous reports (Maren, 1998, 1999b), and the data from the remaining 52 no-shock control subjects have not been published previously.

Behavioral apparatus. Eight identical observation chambers $(30 \times 24 \times 21 \mathrm{~cm}$; MED-Associates Inc., Burlington, VT) were used for both conditioning and contextual fear testing. The chambers were constructed from aluminum (side walls) and Plexiglas (rear wall, ceiling, and hinged front door) and were situated in sound-attenuating cabinets located in a brightly lit and isolated room. The floor of each chamber consisted of 19 stainless steel rods (4 mm in diameter) spaced $1.5 \mathrm{~cm}$ apart (center to center). The rods were wired to a shock source and solid-state grid scrambler (MED-Associates Inc.) for the delivery of footshock unconditional stimuli (USs). A speaker mounted outside a grating in one wall of the chamber was used for the delivery of acoustic conditional stimuli (CSs). The chambers were cleaned with a 5\% ammonium hydroxide solution, and stainless steel pans containing a thin film of the same solution were placed underneath the grid floors before the rats were placed inside. Ventilation fans in each chest supplied background noise (65 dB, A-scale).

Each conditioning chamber rested on a load-cell platform that was used to record chamber displacement in response to each rat's motor activity. To ensure interchamber reliability, each load-cell amplifier was calibrated to a fixed chamber displacement. The 
output of each chamber's load cell was set to a gain (vernier dial, 8) that was optimized for detecting freezing behavior. Load-cell amplifier output $(-10$ to $+10 \mathrm{~V})$ from each chamber was digitized and acquired online using Threshold Activity software (MEDAssociates, Inc.). The absolute values of the load-cell voltages were computed and these absolute values were multiplied by 10 to yield a load-cell activity scale that ranged from 0 to 100 .

During both the conditioning and extinction sessions, each rat's activity was monitored continuously using the data acquisition system described above. For each chamber, loadcell activity was digitized at $5 \mathrm{~Hz}$, yielding one observation per rat every $200 \mathrm{~ms}$ (300 observations/rat/minute). In all experiments, freezing was quantified by computing the number of observations for each rat that had a value less than the freezing threshold (loadcell activity, 5; animals exhibit freezing when load-cell activity is at or below this value; see Maren, 1998, 1999b). To avoid counting momentary inactivity as freezing, an observation was only scored as freezing if it fell within a contiguous group of at least 5 observations that were all less than the freezing threshold. Thus, freezing was only scored if the rat was immobile for at least $1 \mathrm{~s}$. For each session, the freezing observations were transformed to a percentage of total observations. In addition to freezing, motor activity was quantified during the preshock period on the conditioning day using the raw load-cell output.

Surgery. Rats were treated with atropine methyl nitrate $(0.04 \mathrm{mg} / \mathrm{kg}$ body weight), anesthetized with an intraperitoneal injection of Nembutal (sodium pentobarbital, 65 $\mathrm{mg} / \mathrm{kg}$ body weight), and mounted in a stereotaxic apparatus (David Kopf Instruments, Tujunga, CA). The scalp was incised and retracted, and head position was adjusted to place bregma and lambda in the same horizontal plane. Small burr holes ( $2 \mathrm{~mm}$ in diameter) were drilled bilaterally in the skull for the placement of a 28-gauge cannula in the basolateral amygdala ( $3.3 \mathrm{~mm}$ posterior to bregma, $5.0 \mathrm{~mm}$ lateral to the midline). A 10- $\mu$ l Hamilton syringe was mounted in an infusion pump (Harvard Apparatus, South Natick, MA) and connected to the injection cannula with polyethylene tubing. NMDA (20 $\mu \mathrm{g} / \mu \mathrm{l}$; Sigma Chemical Co., St. Louis, MO) in $100 \mathrm{mM}$ phosphate-buffered saline (PBS, $\mathrm{pH}=7.4)$ was infused $(0.1 \mu \mathrm{l} / \mathrm{min}) 8.0 \mathrm{~mm}$ ventral to the brain surface $(0.2 \mu \mathrm{l})$ and $7.5 \mathrm{~mm}$ ventral to the brain surface $(0.1 \mu \mathrm{l})$ for each penetration. Five minutes was allowed after each infusion for diffusion of the drug. Following surgery, the incision was closed with stainless steel wound clips, and the rats were allowed to recover on a heating pad before returning to their home cage. Sham rats were treated similarly, except that cannulas were not lowered into the brain and no drug was infused.

Procedure. Eighty-four rats were randomly assigned to a $2 \times 2$ factorial design with factors of surgery (sham or BLA lesion) and training trials (1 or 75 trials). An additional group of animals was assigned to a group receiving neither surgery nor conditioning. These assignments yielded the following groups: SH-1 $(n=23)$, BL-1 $(n=17)$, SH-75 $(n=10)$, BL-75 $(n=10)$, and NO-SHOCK $(n=24)$. On the conditioning day, the rats were transported to the laboratory in squads of 8 and placed in the conditioning chambers; the chamber position was counterbalanced for each squad and group. All the rats, except for those in the NO-SHOCK group, received unsignaled footshock $(2 \mathrm{~s}, 1.0 \mathrm{~mA} ; 60 \mathrm{~s}$ intertrial interval; 1 or 75 trials) 3 min after being placed in the chambers. Sixty seconds following the final shock, the rats were returned to their home cages. One day after fear conditioning the rats received either sham surgery or BLA lesions. One week following 
recovery from surgery, fear conditioning to the context of the conditioning chamber was assessed by returning the rats to the conditioning chambers and measuring freezing behavior (somatomotor immobility except that necessitated by breathing) during an 8-min extinction test.

I also examined reacquisition of conditional freezing in BLA rats after context extinction testing. For this analysis, the groups consisted of the SH-75 and BL-75 groups described above, as well as SH-0 $(n=12)$ and BL-0 $(n=16)$ groups that were run concurrently with the 75-trial rats in a different experiment. Reacquisition training consisted of 25 additional context-shock trials on each of 2 consecutive days. A 4-min shock-free period occurred before each shock session, and freezing during these 4-min periods served as the measure of conditional fear.

Histology. Histological verification of lesion location was performed after behavioral testing. Rats were perfused across the heart with $0.9 \%$ saline followed by $10 \%$ formalin. After extraction from the skull, the brains were postfixed in $10 \%$ formalin for 2 days and $10 \%$ formalin/30\% sucrose until sectioning. Coronal sections (50 $\mu \mathrm{m}$ thick, taken every $200 \mu \mathrm{m})$ were cut on a cryostat $\left(-16^{\circ} \mathrm{C}\right)$ and wet-mounted on glass microscope slides with $70 \%$ ethanol. After drying, the sections were stained with $0.25 \%$ thionin to visualize neuronal cell bodies. Lesions were verified by reconstructing the damage on stereotaxic atlas templates.

Data analysis. For each session, the freezing data were transformed to a percentage of total observations, a probability estimate that is amenable to analysis with parametric statistics. I also quantified the latency to the first bout of freezing, the total number of freezing bouts, the duration of freezing bouts, and the frequency of bouts of various lengths. These data were analyzed using analysis of variance (ANOVA). Post-hoc comparisons in the form of Newman-Keuls tests were performed following a significant omnibus $F$ ratio. All data are represented as means \pm the standard errors of the means (SEMs).

\section{RESULTS}

\section{Histology}

The neurotoxic BLA lesions are illustrated and described in previous reports (Maren, 1998, 1999b). In general, there was nearly complete damage to the BLA. The central amygdaloid nucleus was largely spared, although there was minimal damage to its most caudal aspect in some cases. Minimal extraamygdaloid damage was evident in the amygdalohippocampal area and the entorhinal cortex. Rats with unilateral or partial bilateral lesions of the BLA were excluded from the statistical analyses (Maren, 1998, 1999b).

\section{Behavior}

The computerized system that my laboratory uses to monitor displacement of the conditioning chamber permits us to assess motor activity and freezing in rats with a high degree of temporal resolution. To illustrate this point, Fig. 1 displays chamber displacement data for three representative rats during the 8-min context extinction test. These data illustrate the high degree of temporal resolution with which motor activity and freezing 
$6.4 \mathrm{~s}$

$6.6 \mathrm{~s}$
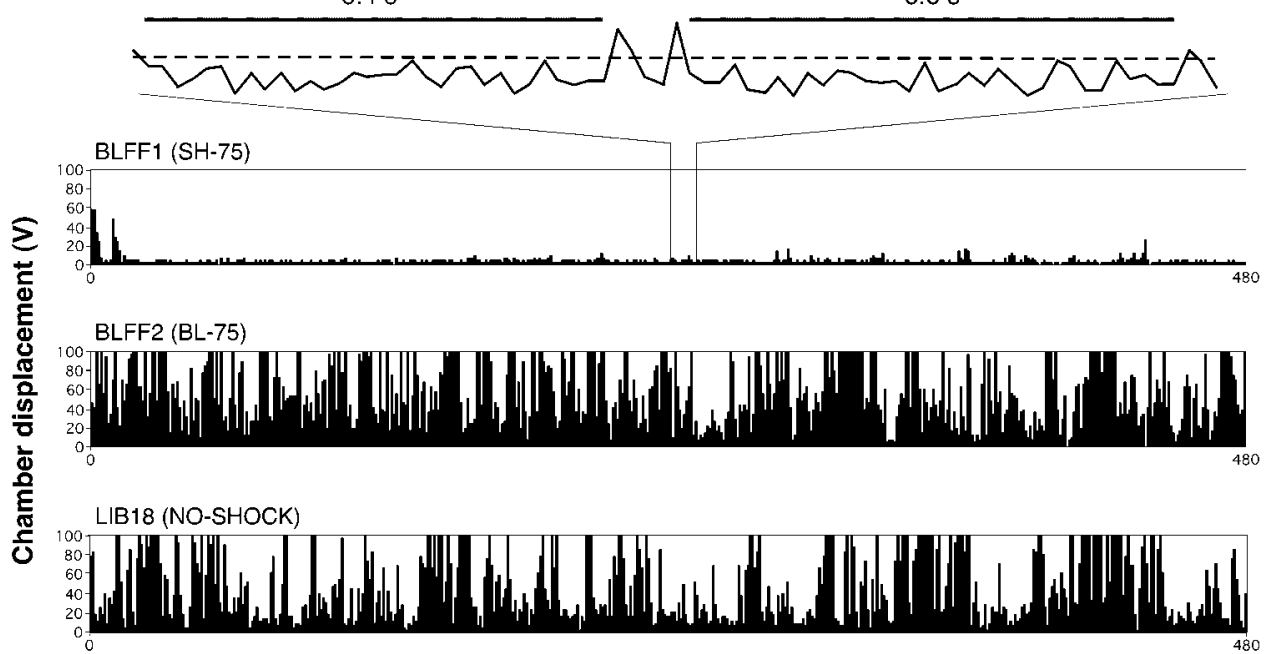

Time (s)

FIG. 1. Chamber displacement data from representative rats in three of the experimental groups ( $\mathrm{SH}-75$, BL-75, and NO-SHOCK). Load-cell voltages (which reflect displacement of the conditioning chambers) were sampled at $5 \mathrm{~Hz}$ (one observation per $200 \mathrm{~ms}$ ) during the course of an 8-min context extinction test. For clarity, the absolute values of these voltages were multiplied by a factor of 10 (the load-cell output ranges from +10 to $-10 \mathrm{~V}$ ). Any contiguous block of five bins with transformed voltages less than 5 were scored as freezing behavior. The inset at the top illustrates two bouts of freezing in the sham rat during a brief epoch midway through the extinction test.

can be assessed with our data acquisition system. The waveform displayed at the top of Fig. 1 illustrates a 15-s epoch of data from a sham rat that received 75 conditioning trials. As described under Materials and Methods, and freezing is scored whenever the animal's activity (indexed by the voltage output of the load-cell amplifiers connected to each chamber) drops below the "freezing threshold," which is indicated by the dashed line (the freezing threshold is equal to a value of 5). Thus, the sham rat displayed in Fig. 1 exhibited two distinct bouts of freezing ( 6.4 and $6.6 \mathrm{~s}$, respectively) during the 15-s epoch that is displayed.

As previously reported (Maren, 1998, 1999b), neurotoxic BLA lesions made 1 day after contextual fear conditioning produced severe retrograde amnesia. This was evident in the severe attenuation in conditional freezing in rats with BLA lesions. As shown in Fig. 1, BLA lesions severely impaired the expression of conditional freezing behavior relative to sham surgery, and the rat receiving BLA lesions did not differ from the NOSHOCK control. Specifically, during the entire 8-min extinction test, the SH-75 rat exhibited 61 bouts of freezing that accounted for $7.1 \mathrm{~min}$ of freezing (88.8\% of the test). The BL-75 and NO-SHOCK rats exhibited 7 and 19 bouts of freezing, respectively, which accounted for $8.6 \mathrm{~s}(1.8 \%)$ and $25.0 \mathrm{~s}(5.2 \%)$ of freezing in these rats.

Figure $2 \mathrm{~A}$ shows average conditional freezing to the training context during an 8-min extinction test for all of the rats in each group. A two-way ANOVA with variables of group (SH-1, SH-75, BL-1, BL-75, and NO-SHOCK) and minute (test minutes 1-8) revealed significant main effects of group $[F(4,79)=36.1, p<.0001]$ and minute $[F(7$, $553)=5.0, p<.0001]$ and a significant interaction between these variables $[F(28,553)$ $=5.4, p<.0001]$. Post-hoc comparisons $(p<.05)$ of the overall group means indicated 

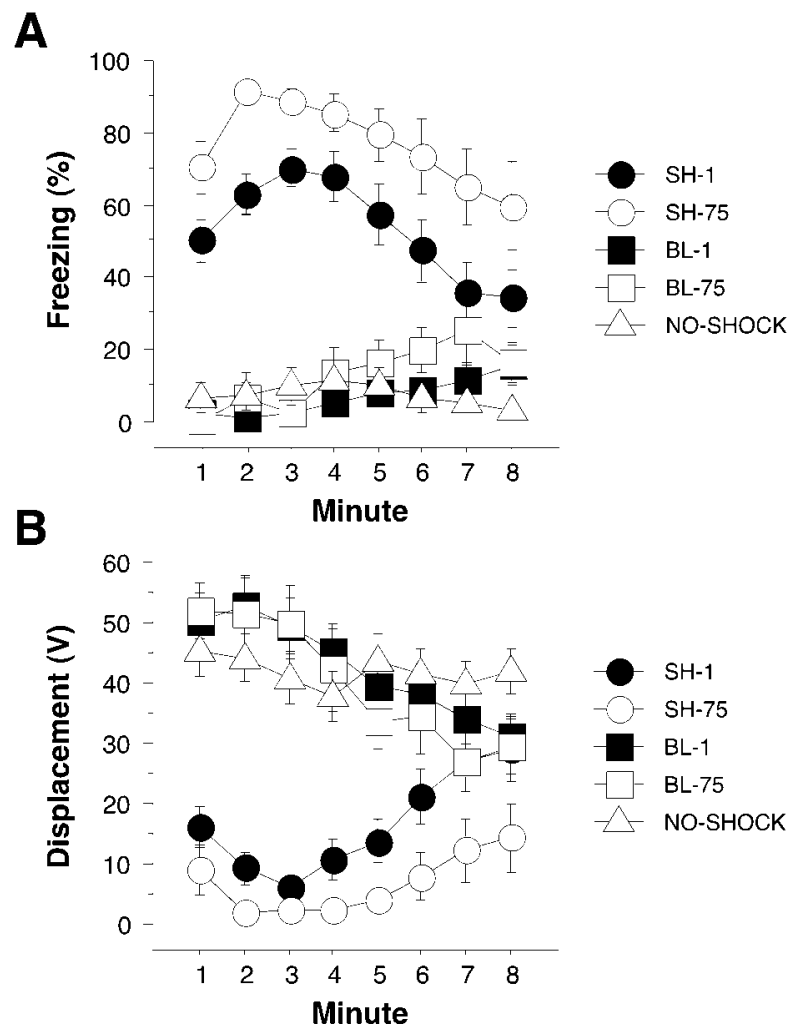

FIG. 2. (A) Mean ( \pm SEM) percentage of freezing and (B) chamber displacement (an index of motor activity) during each minute of an 8-min context extinction test. Rats received either sham (SH) surgery or neurotoxic BLA lesions (BL) $24 \mathrm{~h}$ after either 1 or 75 contextual fear conditioning trials. NO-SHOCK rats received the same amount of exposure to the conditioning boxes as the 75-trial animals, but no footshock was delivered.

that rats with BLA lesions exhibited significantly lower levels of freezing than shamoperated rats and did not differ significantly from each other or from rats that did not receive footshock during training. Moreover, these comparisons indicated that shamoperated rats receiving 75 conditioning trials exhibited significantly more conditional freezing than shams receiving a single conditioning trial. Comparisons of the test minute means revealed the same pattern of results. Importantly, there were no significant differences between rats with BLA lesions and the NO-SHOCK rats at any point during the extinction test.

Another index of contextual fear conditioning that is highly correlated with freezing behavior is suppression of locomotor activity. I examined activity suppression because it may be more sensitive to spared memory in rats with BLA lesions. In other words, BLA rats could show suppression of ongoing activity during extinction testing without freezing. Figure 2B shows average load-cell output (a measure of chamber displacement) during the 8-min extinction test. As with freezing behavior, a two-way ANOVA with variables of group (SH-1, SH-75, BL-1, BL-75, and NO-SHOCK) and minute (test minutes 1-8) revealed significant main effects of group $[F(4,79)=18.5, p<.0001]$ and minute $[F(7$, $553)=3.9, p<.0005]$ and a significant interaction between these variables $[F(28$, $553)=7.9, p<.0001]$. Post-hoc comparisons $(p<.05)$ of the overall group means 
indicated that rats with BLA lesions exhibited significantly lower levels of chamber displacement (i.e., motor activity) than sham-operated rats and did not differ significantly from each other or rats that did not receive footshock during training. Unlike freezing, comparisons of these means did not reveal a difference in motor activity between shamoperated rats receiving 75 conditioning trials and those receiving a single conditioning trial. However, comparisons of the group means during each test minute revealed greater activity suppression in SH-75 than in SH-1 rats in the final $3 \mathrm{~min}$ of the extinction test. Again, there were no differences between rats with BLA lesions and the NO-SHOCK rats at any point during the extinction test. Hence, motor activity is not more sensitive than freezing for detecting impairments in rats with BLA lesions, and it is less effective than freezing in differentiating sham rats receiving 1 or 75 conditioning trials.

To gain further insight into the nature of the freezing impairment in rats with BLA lesions, I performed a more detailed analysis of freezing behavior than is typically reported. This analysis focused on determining the onset and the duration of discrete bouts of freezing behavior to complement the freezing duration data presented in the preceding analyses. My implementation of this detailed analysis was motivated by a report published several years ago by Blanchard and colleagues (Blanchard, Blanchard, Lee, \& Fukunaga, 1977). This report showed that rats with hippocampal lesions freeze less than control rats in a novel environment, but initiate the freezing response more often. This suggests that the initiation and maintenance of freezing behavior are dissociable, and it is conceivable that BLA lesions could affect the latter without affecting the former after fear conditioning. If this pattern of results were obtained, it would suggest that a memory for fear conditioning was present in rats with BLA lesions and that their primary deficit is in the maintenance of the freezing response.

I first considered whether the latency to freeze during extinction testing is affected by posttraining BLA lesions. Figure 3 shows the latency to the first bout of freezing for rats in each of the five groups. It is evident that sham-operated rats froze at a much shorter latency than rats with BLA lesions and that rats with BLA lesions did not differ from rats that did not receive footshock. These impressions were confirmed by a significant

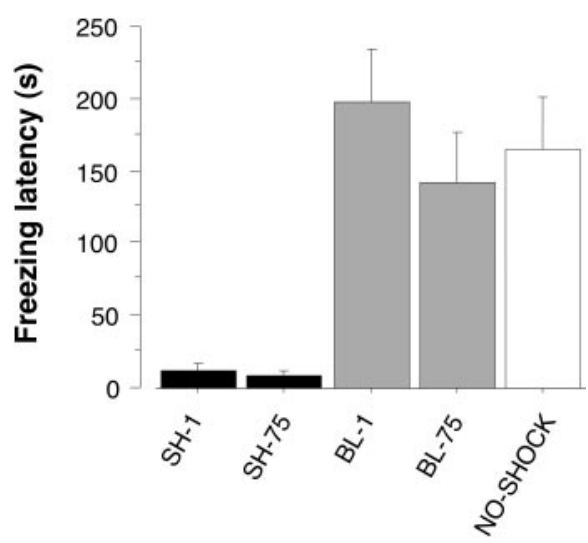

FIG. 3. Mean ( \pm SEM) latency (in seconds) to the first bout of freezing during the 8-min context extinction test. Rats received either sham (SH) surgery or neurotoxic BLA lesions (BL) $24 \mathrm{~h}$ after either 1 or 75 contextual fear conditioning trials. NO-SHOCK rats received the same amount of exposure to the conditioning boxes as the 75-trial animals, but no footshock was delivered. 
main effect of group in a one-way ANOVA performed on these data $[F(4,79)=8.5$, $p<.0001$ ], and post-hoc comparisons $(p<.05)$ confirmed that sham rats differed from rats in all other groups, but did not differ from one another. Moreover, these comparisons indicated that NO-SHOCK rats did not differ from rats with BLA lesions. Hence, on this measure of conditional fear, spared memory in rats with posttraining BLA lesions is not evident.

In addition to examining the latency to the first bout of freezing, I examined the number, duration, and temporal distribution of freezing bouts during extinction testing. Again, I performed this analysis because it is conceivable that BLA lesions produce a selective impairment in the maintenance, as opposed to initiation, of freezing behavior. If BLA lesions do not affect conditional fear memories, then one would observe a greatly reduced freezing duration in these animals without a change (or even an increase) in the number of freezing bouts. Figure 4A displays the number of freezing bouts emitted by rats in each of the five groups during extinction testing, and Fig. 4B shows a cumulative record of these same data. For both data sets, the outcome is familiar. That is, sham rats exhibit significantly more freezing bouts that rats with BLA lesions or NO-SHOCK rats, which do not differ from each other. These observations were confirmed by significant main effects of group [bouts; $F(4,79)=16.5, p<.0001$; cumulative bouts, $F(4,79)=24.2$,
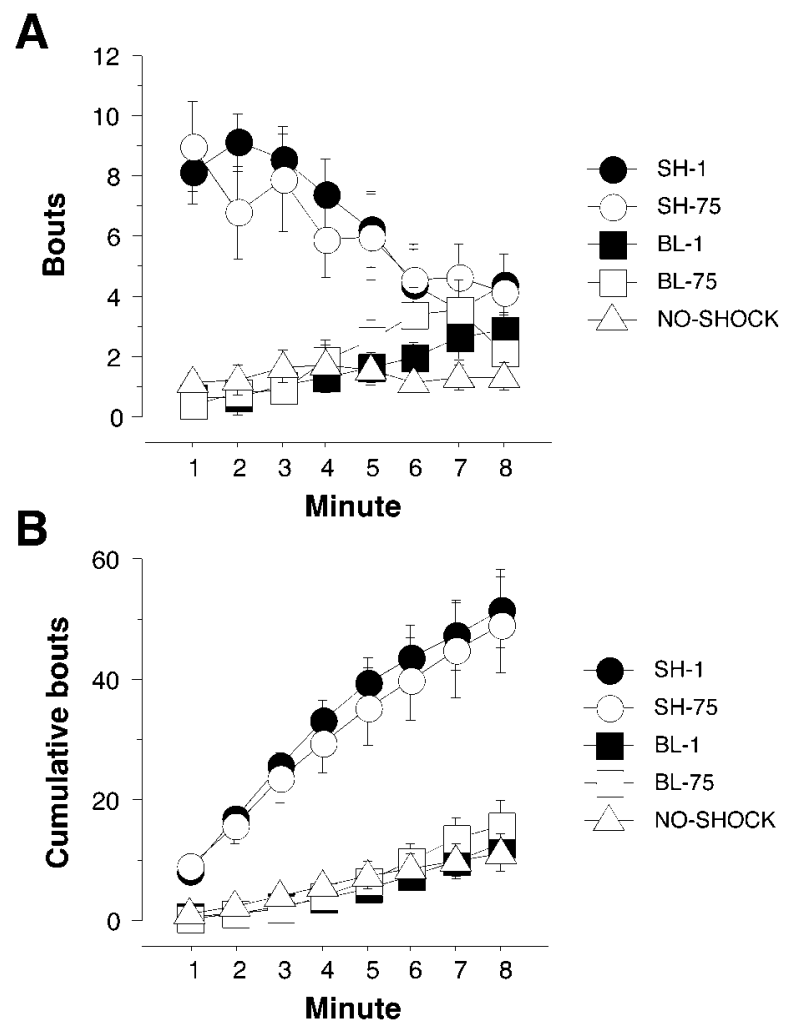

FIG. 4. (A) Mean ( \pm SEM) number of freezing bouts and (B) cumulative number of freezing bouts during each minute of an 8-min context extinction test. Rats received either sham (SH) surgery or neurotoxic BLA lesions (BL) $24 \mathrm{~h}$ after either 1 or 75 contextual fear conditioning trials. NO-SHOCK rats received the same amount of exposure to the conditioning boxes as the 75-trial animals, but no footshock was delivered. 
$p<.0001$ ] and significant group $\mathrm{x}$ minute interactions [bouts, $F(28,553)=5.7, p<$ .0001 ; cumulative bouts, $F(28,553)=97.3, p<.0001]$ in the ANOVAs. Post-hoc comparisons $(p<.05)$ on both the overall group means and the individual data for each test minute indicated that sham rats receiving 1 or 75 trials did not differ from one another. Moreover, BLA rats receiving 1 or 75 trials did not differ from each other or NO-SHOCK controls. Unlike the freezing duration data or the motor activity data, these data do not discriminate between sham rats receiving 1 and 75 trials. Nonetheless, this measure is still robust in its ability to differentiate sham rats from rats with BLA lesions. Interestingly, it does not appear that BLA lesions dissociate the initiation and maintenance of conditional freezing. That is, BLA lesions produced massive deficits in the duration of freezing, the latency to freeze, and the number of freezing bouts.

This analysis necessarily suggests that the difference in freezing duration between SH75 and SH-1 rats reflects longer bouts of freezing (rather than more bouts of freezing) in SH-75 rats. Figure 5 reveals that this is, in fact, the case. Sham-operated rats exhibited longer freezing bouts than BLA rats or NO-SHOCK rats, and SH-75 rats exhibited longer freezing bouts than SH-1 rats. These observations were confirmed by a main effect of group in a one-way ANOVA performed on these data $[F(4,79)=7.4, p<.0001]$ and post-hoc comparisons $(p<.05)$. BL-1 and BL-75 exhibited shorter bouts of freezing than SH-75 rats, but not SH-1 rats; they did not differ from either each other or NOSHOCK rats.

The previous analyses indicate that BLA lesions reduce both the average duration of freezing and the average frequency of these bouts. It is conceivable, however, that my measures of freezing duration and frequency could obscure subtle shifts in the frequency of various "bout lengths." To address this question, I measured the duration of individual bouts of freezing and assigned these bout lengths to bins with 1 of 480 possible values (i.e., bouts could vary in duration from 1 to $480 \mathrm{~s}$ in 1-s increments). I then plotted the number of bouts that occurred during the 8-min extinction test at each possible bout length. These data are shown in Fig. 6 for bouts ranging in duration from 1 to $20 \mathrm{~s}$. I do not show the data for longer bouts of freezing (21-480 s), because these events were rare

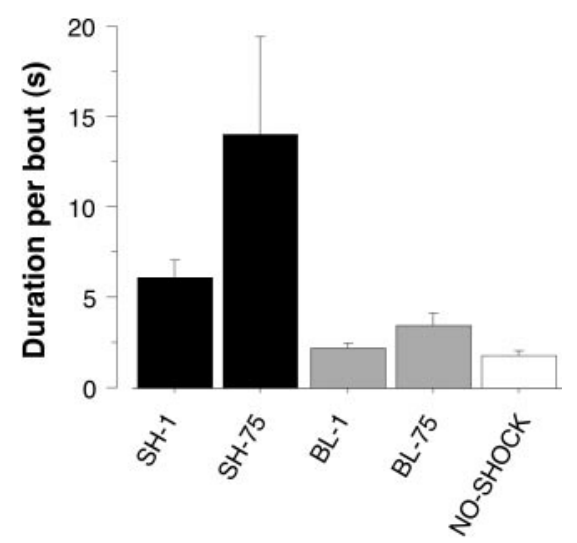

FIG. 5. Mean ( \pm SEM) duration per bout of freezing during the 8-min context extinction test. Rats received either sham (SH) surgery or neurotoxic BLA lesions (BL) $24 \mathrm{~h}$ after either 1 or 75 contextual fear conditioning trials. NO-SHOCK rats received the same amount of exposure to the conditioning boxes as the 75 -trial animals, but no footshock was delivered. 

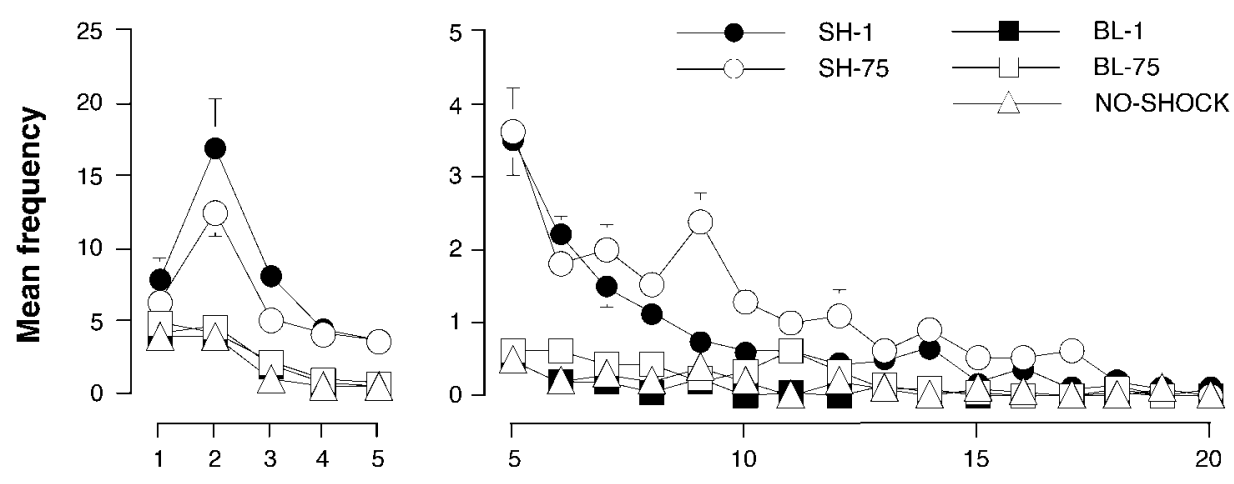

\section{Bout length (1-s bins)}

FIG. 6. Mean ( \pm SEM) frequency of bout lengths (1-s bins) during the 8-min context extinction test. Rats received either sham (SH) surgery or neurotoxic BLA lesions (BL) $24 \mathrm{~h}$ after either 1 or 75 contextual fear conditioning trials. NO-SHOCK rats received the same amount of exposure to the conditioning boxes as the 75-trial animals, but no footshock was delivered.

and did not account for group differences. There are three important conclusions that can be drawn from these data. First, fear conditioning increased the overall frequency of bout lengths, but had a markedly greater affect for some bout lengths than others. Compared to NO-SHOCK controls, for example, the frequency of 2-s bout lengths was augmented to a much greater extent in sham-operated rats than the frequency of 1-s bout lengths, which did not differ among the groups. Second, extensive overtraining was associated with a shift to the right in the frequency distribution of bout lengths. Hence, longer bout lengths (9-, 10-, and 12-s bins) were more frequent, and shorter bout lengths (2- and 3$\mathrm{s}$ bins) less frequent in SH-75 than in SH-1 rats. Third, rats with BLA lesions exhibited a frequency distribution in bout lengths that was comparable to that of NO-SHOCK controls. Importantly, it did not exhibit the same shape (i.e., peak at the 2-s bin) that was evident in sham rats. Collectively, these data indicate that there is not savings for fear memory when it is assessed by freezing duration, latency, or frequency.

As a final test for savings, I compared the rate of reacquisition of conditional freezing in sham and BLA rats receiving either 0 or 75 conditioning trials before lesions and subsequent reacquisition training. I was interested in determining whether rats with BLA lesions that had received 75 fear conditioning trials prior to the lesion would show an accelerated reacquisition rate compared to BLA rats that did not receive fear conditioning prior to their lesion. The results, which are shown in Fig. 7, are unambiguous: BL-0 and BL-75 rats reacquired conditional freezing at nearly identical rates. A three-way ANOVA revealed main effects of lesion $[F(1,44)=24.6, p<.0001]$, trial $[F(1,44)=4.8, p<$ $.05]$, and test period $[F(2,88)=196.3, p<.0001]$ and importantly, a significant interaction between these variables $[F(2,88)=36.2, p<.0001]$. Post-hoc comparisons $(p<.05)$ revealed that $\mathrm{SH}-0$ rats acquired conditional fear at a faster rate than both BL-0 and BL75 rats, which did not differ from one another. All of the groups exhibited similar levels of freezing by the end of reacquisition training consistent with earlier reports (Maren, 1999b). In summary, I have found no evidence for savings of conditional fear memory in rats with posttraining BLA lesions using multiple measure of freezing and motor activity. 


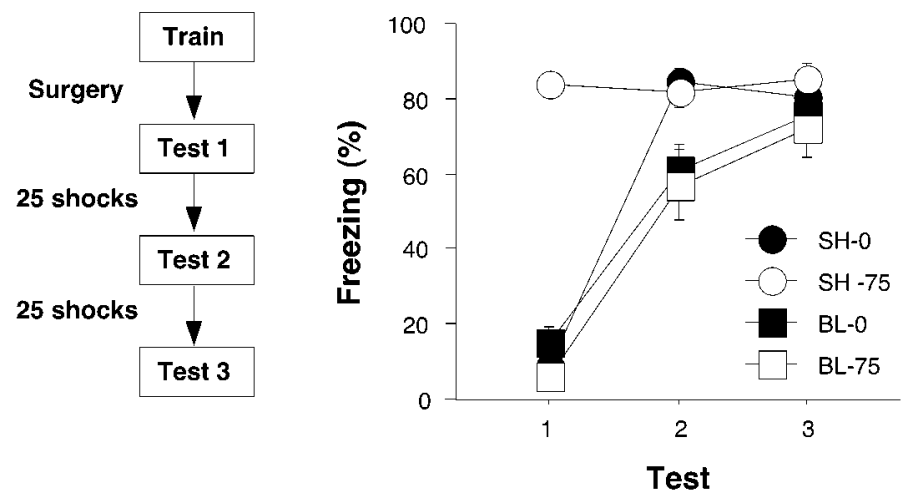

FIG. 7. Mean ( \pm SEM) percentage of freezing during three 4-min context extinction tests conducted at various times relative to original training, surgery, and reacquisition training (see left panel for experimental flow chart). Rats received either sham (SH) surgery or neurotoxic BLA lesions (BL) $24 \mathrm{~h}$ after either 1 or 75 contextual fear conditioning trials. NO-SHOCK rats received the same amount of exposure to the conditioning boxes as the 75-trial animals, but no footshock was delivered.

\section{DISCUSSION}

The present study used a detailed analysis of freezing behavior to examine whether there is spared memory for contextual fear conditioning in rats with posttraining neurotoxic BLA lesions. In addition to quantifying the percentage of freezing during extinction testing behavior (a measure of the total duration of freezing behavior), I quantified the latency to exhibit freezing, the number of bouts of freezing, the average duration of freezing bouts, and the frequency distribution of various bout lengths. The pattern of results for all measures of freezing was similar. Rats with posttraining BLA lesions exhibited severe deficits in conditional fear irrespective of the degree of original training and exhibited a pattern of behavior that was comparable to that of animals that did not receive footshock during training. This reveals that there is not spared memory or "savings" for contextual fear conditioning, at least as it is indexed by conditional freezing, in rats receiving neurotoxic BLA lesions $24 \mathrm{~h}$ following conditioning. These data converge with the results of other studies indicating that many different fear responses, including potentiated acoustic startle (Campeau \& Davis, 1995; Sananes \& Davis, 1992), analgesia (Helmstetter \& Bellgowan, 1993), and changes in heart rate (Gentile, Jarrell, Teich, McCabe, \& Schneiderman 1986), are disrupted by posttraining amygdala lesions. Moreover, these data support the hypothesized role of the BLA in the encoding and storage of fear memories (Davis, 1997; Fanselow \& LeDoux, 1999; LeDoux, 2000; Maren, 1999a, 2001). By this view, posttraining BLA lesions result in an abolition of conditional freezing because they erase the memory for the CS-US association established during conditioning.

The absence of savings in rats with BLA lesions in the present study conflicts with results reported by Cahill et al. (2000). However, there is an important procedural difference between these two reports that accounts for this discrepancy. Whereas I have made BLA lesions after fear conditioning, Cahill and colleagues induced BLA lesions 1 week before fear conditioning. Under these latter conditions, it is likely that savings of fear memory (at least as it is indexed by conditional freezing to a context) was observed because another neural system was recruited to mediate fear conditioning. Consistent with this possibility, I have found that contextual (but not auditory) fear conditioning is acquired, albeit slowly, 
by rats with neurotoxic BLA lesions (Maren, 1999b). Although these data indicate that conditional freezing and fear conditioning (and hence savings of fear memory) can occur in the absence of the BLA, they do not indicate whether the BLA is normally a locus for fear memory or whether fear memory once acquired is dependent on the BLA. In this regard, the present study makes an important contribution because it demonstrates that fear memory, as it is expressed in conditional freezing, normally requires the BLA. That is, posttraining BLA lesions eliminated conditional freezing and did not leave a residual memory that might have promoted savings during subsequent reacquisition training.

Although the present data are consistent with a role for the BLA in associative processes during fear conditioning, there are alternative interpretations for these results. First, it might be argued that the BLA is necessary for the expression of fear memories formed and stored outside of the amygdala (e.g., Cahill et al., 1999). By this view, BLA lesions disrupt the performance of conditional fear responses, such as freezing, rather than the formation or storage of CS-US associations. However, this view seems unlikely insofar as rats with BLA lesions are capable of exhibiting freezing behavior. For example, rats with neurotoxic BLA lesions exhibit near normal unconditional freezing to a synthetic predator odor (Wallace \& Rosen, 2001). Moreover, rats with BLA lesions are capable of expressing high levels of freezing behavior after extensive overtraining (present results; Maren, 1999b). Other data indicate both spared and deficient freezing performance to contextual and acoustic CSs, respectively, in individual rats trained after the induction of BLA lesions (Maren, 1999b). Thus, the severe deficits in conditional freezing exhibited by rats after posttraining BLA lesions are not due to a performance deficit in freezing behavior.

Another possibility is that neurotoxic BLA lesions disrupt fear memory by inducing either aberrant neuronal activity (e.g., seizure) or transsynaptic damage in connected brain structures. By this view, fear memories that reside outside the BLA are subject to disruption by NMDA infusion into the BLA. Further studies are required to examine the consequence of NMDA infusions into the BLA on electrophysiological and histopathological profiles of connected brain structures. Nonetheless, temporary inactivation of BLA neurons, which is not associated with seizure activity or neuronal degeneration, also prevents the expression of conditional fear memories (Maren, Aharonov, Stote, \& Fanselow, 1996b; Muller, Corodimas, Fridel, \& LeDoux, 1997; Wilensky, Schafe, \& LeDoux, 1999). These data suggest that it is disruption of neuronal activity in the BLA, and not connected brain structures, that accounts for deficits in conditional freezing after posttraining BLA lesions.

In the present experiments, BLA lesions were made within $24 \mathrm{~h}$ after fear conditioning. It is possible that the BLA is engaged in the consolidation of fear memories outside the amygdala during this training-to-surgery interval (Cahill \& McGaugh, 1998; McGaugh, 2000). According to this view, disruption of conditional freezing during retention testing results because the BLA lesion disrupted amygdaloid processes necessary for the consolidation of fear memory outside of the amygdala. Two lines of evidence argue against this possibility. First, immediate posttraining infusions of muscimol into the BLA, which reversibly inactivates BLA neurons, do not affect retention of fear memory tested $24 \mathrm{~h}$ after the infusion (Wilensky et al., 1999). In contrast, posttraining infusions of protein synthesis inhibitors or protein kinase inhibitors into the BLA yield consolidation impairments (Schafe \& LeDoux, 2000). Moreover, a pretraining injection of APV into the BLA, which suppresses amygdaloid spike firing (Maren \& Fanselow, 1995), produces a deficit in the acquisition of conditional fear (Maren et al., 1996b; Walker \& Davis, 2000), whereas 
immediate posttraining APV infusions fail to impair conditional freezing (Maren et al., 1996b). The most parsimonious interpretation of these results is that memory consolidation occurs within the amygdala via protein synthesis-dependent and protein kinase-dependent processes and does not require amygdaloid spike firing. Nonetheless, axons passing through the amygdala may be important for memory consolidation, because posttraining lidocaine infusions have been reported to impair the retention of conditional fear (Vazdarjanova \& McGaugh, 1999). Second, the consolidation hypothesis predicts that BLA lesions made a long time after fear conditioning should have a smaller effect on conditional fear memories than lesions made shortly after conditioning. In contrast to this prediction, neurotoxic BLA lesions made up to 1 month after fear conditioning produce a deficit in conditional fear that is of the same magnitude as that produced by lesions made within 24 h of fear conditioning (Lee, Walker, \& Davis, 1996; Maren, Aharonov, \& Fanselow, 1996a). This pattern of results is obtained after both limited training (Maren et al., 1996a) and overtraining (Lee et al., 1996).

If one accepts that BLA lesions do not impair the performance of fear (Maren, 1999b), then these data support the view that posttraining BLA lesions produce deficits in conditional freezing by disrupting the memory for fear conditioning. It is important to note, however, that the timing of BLA lesions with respect to training is a critical determinant of the nature and extent of deficits in conditional fear. Whereas posttraining BLA lesions produce a global and complete deficit in conditional freezing that is independent of either the extent of training or CS modality, pretraining BLA lesions produce a more limited deficit in conditional freezing (present results; Maren, 1998, 1999b). That is, pretraining lesions produce global deficits in auditory fear conditioning even after extensive overtraining, whereas deficits in contextual fear conditioning are overcome by overtraining (Maren, 1999b). Pre- and posttraining lesions also have differential effects when made in other components of the neural circuit underlying fear conditioning. For example, neurotoxic lesions of the dorsal hippocampus also produce much greater deficits in conditional freezing when made after training (Maren, Aharonov, \& Fanselow, 1997).

The presence of conditional freezing in rats with pretraining BLA lesions suggests that an alternate neural system can acquire and express context-US associations in the absence of the BLA. This alternate system requires considerably more training to achieve normal performance than is typical for the BLA (Maren, 1999b), but appears to support some conditioning after relatively few training trials (Cahill et al., 2000). Importantly, this alternate system does not appear to acquire context-US associations in intact rats, insofar as rats with posttraining BLA lesions exhibit no sparing or savings of fear memory as demonstrated in the present study. Hence, the alternate fear conditioning circuit is engaged only after damage to the amygdala and is only capable of acquiring some forms of fear conditioning (i.e., context-US associations).

In conclusion, several lines of evidence suggest that neurotoxic BLA lesions produce associative, rather than performance, deficits in the acquisition and expression of Pavlovian fear conditioning. The present study does not reveal evidence for savings of fear memory in rats with posttraining neurotoxic BLA lesions using several measures of freezing behavior. These results support the hypothesis that fear memory, as it is expressed in freezing behavior, requires neurons in the BLA. Further studies using reversible inactivation techniques are needed to further resolve the distinction between the role of the BLA in learning versus performing fear memories. Moreover, it is important to examine the 
generality of these results using other indices of fear memory, such as avoidance, hypertension, hypoalgesia, and fear-potentiated startle.

\section{REFERENCES}

Blanchard, D. C., Blanchard, R. J., Lee, E. M. C., \& Fukunaga, K. K. (1977). Movement arrest and the hippocampus. Physiological Psychology, 5, 331-335.

Cahill, L., \& McGaugh, J. L. (1998). Mechanisms of emotional arousal and lasting declarative memory. Trends in Neuroscience, 21, 294-249.

Cahill, L., Vazdarjanova, A., \& Setlow, B. (2000). The basolateral amygdala complex is involved with, but is not necessary for, rapid acquisition of Pavlovian 'fear conditioning'. European Journal of Neuroscience, 12, 3044-3050.

Cahill, L., Weinberger, N. M., Roozendaal, B., \& McGaugh, J. L. (1999). Is the amygdala a locus of "conditioned fear"? Some questions and caveats. Neuron, 23, 227-228.

Campeau, S., \& Davis, M. (1995). Involvement of the central nucleus and basolateral complex of the amygdala in fear conditioning measured with fear-potentiated startle in rats trained concurrently with auditory and visual conditioned stimuli. Journal of Neuroscience, 15, 2301-2311.

Davis, M. (1997). Neurobiology of fear responses: The role of the amygdala. Journal of Neuropsychiatry \& Clinical Neurosciences, 9, 382-402.

Fanselow, M. S., \& LeDoux, J. E. (1999). Why we think plasticity underlying Pavlovian fear conditioning occurs in the basolateral amygdala. Neuron, 23, 229-232.

Fendt, M., \& Fanselow, M. S. (1999). The neuroanatomical and neurochemical basis of conditioned fear. Neuroscience and Biobehavioral Reviews, 23, 743-760.

Gentile, C. G., Jarrell, T. W., Teich, A., McCabe, P. M., \& Schneiderman, N. (1986). The role of amygdaloid central nucleus in the retention of differential Pavlovian conditioning of bradycardia in rabbits. Behavioral Brain Research, 20, 263-273.

Helmstetter, F. J., \& Bellgowan, P. S. (1993). Lesions of the amygdala block conditional hypoalgesia on the tail flick test. Brain Research, 612, 253-257.

Kim, M., \& Davis, M. (1993). Electrolytic lesions of the amygdala block acquisition and expression of fearpotentiated startle even with extensive overtraining but do not prevent reacquisition. Behavioral Neuroscience, 107, 580-595.

LeDoux, J. E. (2000). Emotion circuits in the brain. Annual Review of Neuroscience, 23, 155-184.

Lee, Y., Walker, D., \& Davis, M. (1996). Lack of a temporal gradient of retrograde amnesia following NMDAinduced lesions of the basolateral amygdala assessed with the fear-potentiated startle paradigm. Behavioral Neuroscience, 110, 836-839.

Maren, S. (1998). Overtraining does not mitigate contextual fear conditioning deficits produced by neurotoxic lesions of the basolateral amygdala. Journal of Neuroscience, 18, 3088-3097.

Maren, S. (1999a). Long-term potentiation in the amygdala: A mechanism for emotional learning and memory. Trends in Neurosciences, 22, 561-567.

Maren, S. (1999b). Neurotoxic basolateral amygdala lesions impair learning and memory but not the performance of conditional fear in rats. Journal of Neuroscience, 19, 8696-8703.

Maren, S. (2001). Neurobiology of Pavlovian fear conditioning. Annual Review of Neuroscience, 24, 897-931.

Maren, S., Aharonov, G., \& Fanselow, M. S. (1996a). Retrograde abolition of conditional fear after excitotoxic lesions in the basolateral amygdala of rats: Absence of a temporal gradient. Behavioral Neuroscience, 110, $718-726$.

Maren, S., Aharonov, G., \& Fanselow, M. S. (1997). Neurotoxic lesions of the dorsal hippocampus and Pavlovian fear conditioning in rats. Behavioural Brain Research, 88, 261-274.

Maren, S., Aharonov, G., Stote, D. L., \& Fanselow, M. S. (1996b). N-methyl-D-aspartate receptors in the basolateral amygdala are required for both acquisition and expression of conditional fear in rats. Behavioral Neuroscience, 110, 1365-1374. 
Maren, S., \& Fanselow, M. S. (1995). Synaptic plasticity in the basolateral amygdala induced by hippocampal formation stimulation in vivo. Journal of Neuroscience, 15, 7548-7564.

McGaugh, J. L. (2000). Memory-A century of consolidation. Science, 287, 248-251.

Muller, J., Corodimas, K. P., Fridel, Z., \& LeDoux, J. E. (1997). Functional inactivation of the lateral and basal nuclei of the amygdala by muscimol infusion prevents fear conditioning to an explicit conditioned stimulus and to contextual stimuli. Behavioral Neuroscience, 111, 683-691.

Parent, M. B., Tomaz, C., \& McGaugh, J. L. (1992). Increased training in an aversively motivated task attenuates the memory-impairing effects of posttraining $N$-methyl-D-aspartate-induced amygdala lesions. Behavioral Neuroscience, 106, 789-797.

Sananes, C. B., \& Davis, M. (1992). N-Methyl-D-aspartate lesions of the lateral and basolateral nuclei of the amygdala block fear-potentiated startle and shock sensitization of startle. Behavioral Neuroscience, 106, $72-80$.

Schafe, G. E., \& LeDoux, J. E. (2000). Memory consolidation of auditory Pavlovian fear conditioning requires protein synthesis and protein kinase A in the amygdala. Journal of Neuroscience, 20, RC96.

Vazdarjanova, A., \& McGaugh, J. L. (1998). Basolateral amygdala is not critical for cognitive memory of contextual fear conditioning. Proceedings of the National Academy of Sciences USA, 95, 15003-15007.

Vazdarjanova, A., \& McGaugh, J. L. (1999). Basolateral amygdala is involved in modulating consolidation of memory for classical fear conditioning. Journal of Neuroscience, 19, 6615-6622.

Walker, D. L., \& Davis, M. (2000). Involvement of NMDA receptors within the amygdala in short- versus long-term memory for fear conditioning as assessed with fear-potentiated startle. Behavioral Neuroscience, 114, 1019-1033.

Wallace, K. J., \& Rosen, J. B. (2001). Neurotoxic lesions of the lateral nucleus of the amygdala decrease conditioned fear but not unconditioned fear of a predator odor: Comparison with electrolytic lesions. Journal of Neuroscience, 21, 3619-3627.

Weinberger, N. M. (1998). Physiological memory in primary auditory cortex: Characteristics and mechanisms. Neurobiology of Learning and Memory, 70, 226-251.

Wilensky, A. E., Schafe, G. E., \& LeDoux, J. E. (1999). Functional inactivation of the amygdala before but not after auditory fear conditioning prevents memory formation. Journal of Neuroscience, 19, RC48. 